\title{
Alterstice
}

Revue internationale de la recherche interculturelle

International Journal of Intercultural Research

Revista International de la Investigacion Intercultural

\section{Expérience d'immigration et droit à la santé, à des soins et à des services de qualité : une question de justice sociale}

\section{Bilkis Vissandjée, Karine Bates, François Vialla et Jonathan Kuntz}

Volume 3, numéro 1, 2013

URI : https://id.erudit.org/iderudit/1077500ar

DOI : https://doi.org/10.7202/1077500ar

Aller au sommaire du numéro

Éditeur(s)

Alterstice

ISSN

1923-919X (numérique)

Découvrir la revue

Citer cet article

Vissandjée, B., Bates, K., Vialla, F. \& Kuntz, J. (2013). Expérience d’immigration et droit à la santé, à des soins et à des services de qualité : une question de justice sociale. Alterstice, 3(1), 67-83. https://doi.org/10.7202/1077500ar

\section{Résumé de l'article}

Cette contribution propose une réflexion sur les défis associés au droit à l'accès à des soins de qualité dans une perspective de justice sociale pour les personnes en situation d'immigration. Le but de cet article est de mettre en exergue certains défis de prise en compte de l'interaction de diverses influences lorsqu'il s'agit d'arrimer la question du droit et de la justice sociale à celle de l'accès aux services et soins de santé. Le principe juridique de droit à la protection de la santé ne peut devenir effectif qu'à la condition que l'énoncé d'un droit soit accompagné de moyens concrets de mise en oeuvre et de suivi d'un réseau de distribution de soins efficace pour tous. En ce sens, l'accès à la santé pose des défis similaires à ceux de l'accès à la justice : ils sont souvent tributaires d'une forme d'institutionnalisation imbriquée à des approches trop bureaucratiques, au contraire d'une approche holistique qui viserait à saisir les attentes des personnes requérant des soins et des services. L'accès à des soins de santé, en tant que droit, pourrait être amélioré par la mise en place d'un environnement où un dialogue et une reconnaissance mutuelle seraient réalisés entre sujets de droit et intervenants du système de santé. 


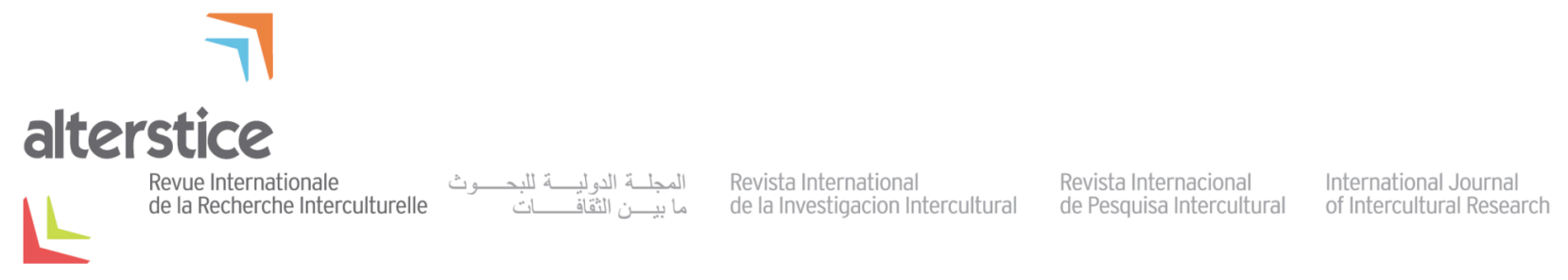

ARTICLE HORS THÈME

\section{Expérience d'immigration et droit à la santé, à des soins et à des services de qualité : une question de justice sociale}

Bilkis Vissandjée ${ }^{1}$, Karine Bates ${ }^{1}$, François Vialla ${ }^{2}$ et Jonathan Kuntz ${ }^{3}$

\section{Résumé}

Cette contribution propose une réflexion sur les défis associés au droit à l'accès à des soins de qualité dans une perspective de justice sociale pour les personnes en situation d'immigration. Le but de cet article est de mettre en exergue certains défis de prise en compte de l'interaction de diverses influences lorsqu'il s'agit d'arrimer la question du droit et de la justice sociale à celle de l'accès aux services et soins de santé. Le principe juridique de droit à la protection de la santé ne peut devenir effectif qu'à la condition que l'énoncé d'un droit soit accompagné de moyens concrets de mise en œuvre et de suivi d'un réseau de distribution de soins efficace pour tous. En ce sens, l'accès à la santé pose des défis similaires à ceux de l'accès à la justice : ils sont souvent tributaires d'une forme d'institutionnalisation imbriquée à des approches trop bureaucratiques, au contraire d'une approche holistique qui viserait à saisir les attentes des personnes requérant des soins et des services. L'accès à des soins de santé, en tant que droit, pourrait être amélioré par la mise en place d’un environnement où un dialogue et une reconnaissance mutuelle seraient réalisés entre sujets de droit et intervenants du système de santé.

\section{Rattachement des auteurs}

${ }^{1}$ Université de Montréal, Montréal, Canada; ${ }^{2}$ Université de Pau et Centre d'Études Droit et santé, Université Montpellier 1, France; ${ }^{3}$ Université de Sherbrooke, Sherbrooke, Canada

\section{Correspondance}

bilkis.vissandjee@umontreal.ca

\section{Mots clés}

droit à la santé; justice sociale; migration; genre; accès aux services de santé

\section{Pour citer cet article :}

Vissandjée, B., Bates, K., Vialla, F. et Kuntz, J. (2013). Expérience d'immigration et droit à la santé, à des soins et à des services de qualité : une question de justice sociale. Alterstice, 3(1), 67-83. 
Porter sa vue au loin. J.-J. Rousseau.

\section{Introduction et cadre d'analyse}

Donner des soins de qualité à tous et à toutes, se donner les moyens pour le faire, exige des professionnels de la santé des stratégies et des pratiques fondées sur une série d'assises, notamment sur la reconnaissance de I'influence de déterminants multiples pour une condition donnée, sur l'examen des capacités à établir des rapports égalitaires et sur la reconnaissance de la complexité à l'établissement de ces derniers (Karlsen et Nazroo, 2002; Le Bossé, 2004; Legault et Rachédi, 2008; Vissandjée et Maillet, 2007). Cette perspective, qui est celle que nous avons choisie ici, tient compte du fait que les disparités entre les personnes, souvent produites par des mouvements socialement ancrés, s'expriment dans le vécu quotidien des femmes et des hommes.

Afin de comprendre les dynamiques et les interactions qui favorisent l'accès tant aux services de santé qu'à la justice d'une personne vivant des expériences d'immigration, il est ainsi nécessaire de circonscrire ses trajectoires, les situations de vulnérabilité rencontrées et ses capacités de résilience. C'est sur cette toile de fond que seront présentés les défis associés au droit à l'accès à des soins de qualité dans une perspective de justice sociale.

L'originalité des réflexions proposées ici est ancrée dans cet arrimage des questions du droit et de la justice sociale aux questions de santé en contexte d'immigration. C'est en effet par la construction d'un pont entre les disciplines que pourront être mieux dégagées les fragilités systémiques qui pavent ces chemins d'accès.

\section{Justice sociale}

Dans le cadre de notre analyse relative aux conditions de vulnérabilité affectant l'accès aux services de santé et au droit à la santé, nous adoptons la définition de la justice sociale proposée par les Nations Unies. La justice sociale est une condition fondamentale de la coexistence pacifique et prospère des femmes et des hommes au sein des nations et entre les nations elles-mêmes. La justice sociale est en cause lorsqu'il s'agit de s'inscrire dans une perspective visant l'égalité des sexes ou les droits des peuples autochtones et des migrants (Organisation internationale du travail, 2008) ${ }^{1}$.

\section{Santé et déterminants sociaux de la santé}

La santé d'une population dépend de l'organisation de conditions matérielles quotidiennes, des déterminants sociaux de la santé, profondément liés aux fondements de l'existence et à la vie économique et sociale dans son ensemble (Santé Canada, 2000; Cooper, 2002; Mikkonen et Dennis, 2011). La santé est un atout indéniable, une ressource essentielle contribuant au développement humain et ayant un effet bénéfique pour l'ensemble d'une société, ce qui est inscrit dans la Constitution de l’Organisation mondiale de la santé (OMS) de 1946.

\section{Vulnérabilité}

S’agissant des questions relatives à la vulnérabilité, c'est dans la perspective des déterminants sociaux de la santé que nous situons nos réflexions. Le contexte de vie, l'environnement comme les expériences de santé lors de l'intégration dans une société nouvelle peuvent présenter un certain nombre de facteurs de risque pour la santé des femmes, des hommes et des familles. La vulnérabilité étant un processus complexe, notre intention ici n'est pas de modéliser. Certains auteurs ont déjà discuté de l'imbrication complexe des interactions reliant la bonne santé à la détérioration du statut de santé, aux facteurs individuels et aux facteurs contextuels. De plus, alors que des modélisations des interdépendances intrinsèques à la diversité des déterminants sociaux de la santé ont été tentées, l'angle de la valeur ajoutée reliée à l'unicité de l'influence de chaque facteur a aussi été captée. Tous soulignent, depuis un certaine nombre d'années, l'importance de la prise en compte de l'influence multiple des déterminants sociaux dans la perspective de singulariser des situations de vie extrêmes mais également d’inscrire

\footnotetext{
${ }^{1}$ « Alors que nous cherchons à construire le monde que nous voulons, redoublons d'efforts pour parvenir à un développement durable plus équitable, qui ne fasse pas de laissés-pour-compte et qui repose sur le dialogue, la transparence et la justice sociale ». Ban Ki-moon, Secrétaire général de l'ONU, 20 février 2013.
} 
les trajectoires de vie dans les dynamiques structurelles qui les ont produites (Bouchard, 2003; Evans, Barer et Marmor, 1996; Le Blanc, Raynault et Lessard, 2012). Une telle prise en compte permet de mettre en relation les circonstances vécues différemment par diverses personnes et collectivités faisant en sorte que certaines sont en meilleure santé que d'autres. En effet, la position sociale de la personne est directement reliée à ses capacités d'accès aux connaissances lui permettant de mettre en œuvre son droit d'accès à des soins de santé de qualité.

\section{Genre}

Les rapports de genre représentent un excellent exemple d'un produit de socialisation suggérant des comportements, des rôles, des droits et des responsabilités faisant partie intégrante de l'ensemble des droits de chaque personne. Des résultats de recherche et certaines jurisprudences attestent par exemple des difficultés de certaines communautés à accepter des soignants de sexe opposé dans la société d’accueil (Gravel, 2000; Vissandjée et Battaglini, 2010).

Alors que le sexe biologique demeure constant, les questions et rapports de genre sont en perpétuelle mouvance au gré des définitions sociales qui leur sont données, influençant l'accès aux ressources et leur utilisation, mettant en exergue les vulnérabilités spécifiques aux femmes et aux hommes (Hankivsky, 2005; Spitzer, 2005; Tingen, Kim, Wu et Woodruff; 2010). En matière de genre, les catégories juridiques sont généralement fondées sur une certaine " vérité " ou " concordance » entre sexe biologique - apparent - et " sexe juridique ", dans une perspective de droit. II faut noter que, dans les cas de réassignation sexuelle - qui permet une apparente conformité entre les sexes physique et psychosocial - le droit ne prive pas le sujet d'une possibilité de reconnaître un changement d'état. Dès lors, puisque le droit accepte une " entorse " à la " vérité » biologique, il semble juste qu'il aille au-delà et accepte un changement d'identité en dépit des conséquences éthiques et sociales (Lawrence et Rieder, 2007; Mateu, Reyner et Vialla, 2000).

\section{Corps en migration}

Le corps en migration participe également à la construction identitaire d'un homme ou d'une femme, de l'âge, du genre et de la classe sociale d'un individu. Ce corps devient indicateur de hiérarchies, de domination et, dans une certaine mesure, emblème de la revendication de certains droits (Lalonde, 2011; Lawrence et Rieder, 2007). C'est ainsi que, exposé ou ignoré, en migration, le corps devient un dialogue, un échange avec soi-même et avec l'autre, les éléments du dialogue provenant d'une mixité de disciplines, de " connaissances formelles ", avec l'intégration d'analyses logiques, de procédures et de pratiques de vie et avec également le souci d'éviter les risques de réduction au corps sans considération d'une certaine marge d'action, et notamment lorsque ce corps est soumis à des jeux d'influence, particulièrement présents lors d'un recours au système de santé ou au système judiciaire (Crenn, 2000; Hubert, 2004; Pietrantonio, 2000).

\section{Mouvance des territoires sociaux}

Une situation de vulnérabilité évolue, puisqu'elle se trouve sans cesse à la croisée d'identités, d'un " territoire social " à un autre, où divers espaces et ressources se conjuguent. Ceci crée un contexte où les rapports sociaux d'inclusion et d'exclusion sont en évolution constante, se constituant et se reconstituant en permanence dans des espaces où chacun et chacune n'est pas de manière égale "sujet de droit " (Carrier, 2008; Gulliford et collab., 2002; Lalonde, 2011; Macall, 2012; Macall, Tremblay et Le Goff, 1997; Mateu, Reyner et Vialla, 2000). De plus, la mouvance de la démographie, de la dynamique des populations et des identités à travers les sociétés exigent que les programmes en matière de santé et de justice s'ajustent sans cesse aux changements conjoncturels, dévoilant des besoins diversifiés soumis à des enjeux sociaux et de droit d'accès.

C'est ainsi qu'élaborer des programmes afin de contribuer à la santé des membres d'une société, dans une perspective de justice sociale, requiert une analyse sensible aux contextes et aux trajectoires d'accès à l'information sur les systèmes en question, car c'est en grande partie l'accès à l'information qui permet de bénéficier ensuite de soins de santé de qualité (Sen, 2000a; Sen, 2000b). 


\section{Droit à la justice, droit à la santé et protection de la santé}

La santé s'inscrit dans le registre de la justice, contribuant directement à la distribution des opportunités dans une société (Anand, Peter, et Sen, 2004; Sen, 2000a). Dans une société où l'accès aux soins et services de santé est légiféré comme devant être d'un accès universel, ce dernier devient ainsi un droit à la protection de la santé au même titre que le droit à la justice.

L'accès à la santé pose des défis similaires à ceux de l'accès à la justice de manière plus globale. Ceci est lié à une forme d'institutionnalisation imbriquée dans une approche bureaucratique et technique qui n'adopte pas d'approche holistique, susceptible pourtant de mieux saisir l'ensemble des besoins et des attentes exprimés par les personnes requérant des soins et des services (Relis, 2009). L'accès à des soins de santé, en tant que droit, peut être amélioré par la mise en place d'un environnement où de réels dialogues s'effectuent entre les sujets de droit et les divers intervenants du système de santé. C'est ainsi que l'effectivité du droit à la protection de la santé pourrait être davantage assuré. Dans l'analyse de l'effectivité de la norme juridique, il s'agit de constater si la norme a provoqué le comportement social prévu. L'effectivité du droit permet d'évaluer l'adéquation et la conformité de la règle de droit en vigueur avec les comportements sociaux des destinataires. La norme juridique est donc effective lorsque les destinataires accomplissent l'obligation édictée par la loi, mais également lorsque les autorités responsables de son application l'exécutent.

Ainsi, le principe juridique de droit à la protection de la santé ne peut devenir effectif qu'à la condition que l'énoncé d'un droit soit accompagné de moyens concrets de mise en œuvre et de suivi d'un réseau de distribution de soins efficace pour tous.

\section{Principaux obstacles à l'exercice des droits et à la justice}

Le droit est un construit qui s'est édifié à partir de catégories et de concepts nés de la vie sociale et en elle; ces catégories et concepts coïncident avec des structures sociales réelles qui, comme les catégories et les concepts, sont constamment en mouvement afin de répondre aux changements sociaux. Le droit n'est pas que normatif; il reflète aussi une certaine perception de la réalité sociale : il a donc une autonomie relative. Une telle autonomie n'est cependant que relative dans la mesure où le droit tire son inspiration des us et coutumes d'une société. Sa production et sa mise en application sont constamment soumises à des jeux de pouvoir dans une société donnée, son action s'inscrit dans les fonctions de diverses institutions variées et, enfin, l'autonomie se nourrit de valeurs et d'idéologies sur lesquelles toute société se construit, se maintient et se modifie (Aubert, 2007; Dion et Breton, 1997; Vissandjée et Maillet, 2007).

La production du droit se déploie donc au cœur d'un champ de valeurs, de codes culturels propres à chaque société, qui lui servent de support interprétatif et dont peuvent découler un certain nombre de contraintes pour son application et son effectivité (Lajoie, 2004, p. 85). Par ailleurs, la reconnaissance de l'ancrage culturel des concepts de droit signifie que l'aspect normatif du droit est le produit d'une interprétation de la justice dans un contexte donné et que le droit est le fruit d'une trajectoire historique complexe. Plusieurs concepts de droit contemporains (tel que l'égalité) ont été articulés au Siècle des Lumières. Cette période fut marquée par la place centrale qu'a commencé à prendre la notion de rationalité dans la société, une rationalité ayant le potentiel d'être objective. Dans le domaine de la science, cette perspective a ouvert la possibilité de prétendre à la compréhension du réel comme un fait objectif pouvant être analysé comme extérieur au contexte socio-politique. Dans le domaine du droit, cette perspective a facilité le renforcement d'une vision positiviste du droit, où une interprétation objective des faits devient envisageable - du moins sur le plan conceptuel. De plus, la présomption de l'existence universelle de certains droits combinée à une bureaucratie juridique en tant que partie intégrante de l’État moderne a engendré un langage offrant un nouvel ensemble de termes, donc un nouveau registre de significations à donner à des mots ou à des expressions courantes. Par conséquent, apprendre le langage juridique s'est dès lors apparenté à un processus d'apprentissage d'un jargon souvent inaccessible aux personnes dites vulnérables (Conley et O'Barr, 1998; Mertz, 2007; Zanchetta et Poureslami, 2006). En ce sens, la culture institutionnelle médicale est similaire à la bureaucratie juridique.

Alterstice - Revue Internationale de la Recherche Interculturelle, vol. 3, $n^{\circ} 1$ 
Un des aspects inégalitaires dans l'acquisition de la connaissance des droits réside dans le fait que le citoyen doit (en tout ou en partie) s'en remettre à la connaissance de spécialistes pour comprendre le système de droit et mettre en œuvre son statut de sujet de droit. Par conséquent, le sujet de droit devient assujetti à une culture professionnelle qui ne lui est pas familière. Pour que le principe d'accès universel à la justice devienne une réalité, le citoyen doit paradoxalement faire appel à des spécialistes qui vont décrypter pour lui le langage du droit (Gravel et collab., 2010). Ainsi, les différents intervenants, tant dans le domaine de la santé que dans celui du droit, deviennent inévitablement des interprètes et des traducteurs. Il est de leur responsabilité d'aider les usagers ou sujets de droit à comprendre les démarches à suivre pour s'assurer d'un accès adéquat tant au système de santé qu'à celui de la justice.

Dans cet ordre d'idées concernant les obstacles qui limitent l'accès à la bureaucratie juridique étatique, il s'avère, d'une part, que les procédures juridiques créent autant d'obstacles, sinon plus, que ceux rencontrés sur le chemin menant à l'accès aux soins de santé. Le droit est alors dévoyé de son but réel qui est d'offrir un cadre au fait social. II n'est plus moyen de régulation, mais instrument d'exclusion. De la même façon, les acteurs du système de santé ne sont pas nécessairement bien formés ni informés pour empêcher que le processus de réclamation de soins de santé ne devienne une source d'obstacles pour le sujet de droit. Ce constat se double d'un abord " scientiste » de la médecine entrée dans l'ère de l'équipe et de l'équipement. L'écran technique mis entre soignants et personnes requérant des soins opacifie la relation au point de rendre diffuse la confiance (cum fides). Le soignant se rassure par l'utilisation d'éléments objectifs et scientifiques (aide à la décision, guides cliniques, scores d'évaluation pour la douleur) mais la relation risque de se déshumaniser. Se trouve ici un parallèle avec une application bureaucratique du droit qui ferait des sujets de droit des cas à gérer en tant que faits objectifs, oubliant que ceux-ci sont des êtres de chair et de sang dotés d'une dimension sociopolitique.

Parmi ces obstacles quant à l'accès à la justice, on retrouve également les coûts directs relatifs à l'obtention de documents officiels (actes notariés, preuves d'identité, de citoyenneté ou d'emploi, etc.), de frais de consultation avec des professionnels du droit, lesquels s'additionnent aux heures de travail perdues, au stress provoqué par la nature complexe des procédures et par les rapports avec les acteurs du milieu juridique (Dembe, Belton, Himmelstein et Workers Compensation Health, 2003; Gravel et collab., 2007). De tels obstacles diminuent les chances qu'une loi ou un droit soit effectif. Les règles formelles d'accès au droit côtoient aussi des pratiques plus informelles vers l'espoir d'augmenter les chances d'avoir gain de cause. Conley et O'Barr (1998) ainsi que Bates (2006), démontrent à cet effet qu'une personne ayant une capacité d'organiser son expérience d' 'injustice » de manière chronologique et sans la teinter de trop d'émotions sera plus facilement entendue par les juges puisqu'elle sera considérée comme cohérente et donc, empreint d'une certaine objectivité. Un tel langage tant verbal que corporel ne fait pas généralement partie des premiers acquis de nouveaux arrivants, qui se retrouvent trop rapidement dans des situations de vulnérabilité.

Ceci revient donc à dire que pour avoir accès à son droit à la justice dans un système prônant le principe d'accès universel à la justice, un citoyen doit, en partie ou entièrement, remettre le contrôle de l'exercice de son droit à celui ou celle sachant décrypter ce jargon juridique, risquant de devenir assujetti à une culture professionnelle étrangère.

\section{Pistes de solutions : promouvoir la littératie en droit et en santé}

Afin que le sujet de droit ait plus de contrôle sur le processus bureaucratique qu'il entreprend pour réclamer ses droits, dont le droit à la santé, il devient nécessaire d'établir des conditions pour permettre un dialogue entre le sujet de droit et la bureaucratie juridique. La place centrale du dialogue est bien souvent oubliée puisque l'aspect normatif du droit est généralement mis de l'avant. Par exemple, la jurisprudence est un processus de rédaction qui permet seulement la transcription de certaines parties des interactions qui ont lieu pendant les procès: les dialogues durant toute la démarche, teintés par un ensemble d'échanges d'idéaux construisant la réalité de la personne requérant une aide juridique, ne sont que peu souvent pris en compte (Bates, 2007; Gravel et collab., 2010). De la sorte, les interactions dans un contexte d'accès à la santé sont trop souvent marquées par des thèmes techniques et un jargon médical méconnu du public. Ces quelques exemples pointent vers une réflexion émise par 
Henri Lacordaire ${ }^{2}$ au $19^{\mathrm{e}}$ siècle, souvent cité par les anti-libéraux, soulignant que le cadre et l'encadrement par la norme permettent de dépasser l'affirmation de principes qui demeureraient lettre morte si l'on ne veillait pas à leur effectivité : « Entre le fort et le faible, entre le riche et le pauvre, entre le maître et le serviteur, c'est la liberté qui opprime et la loi qui affranchit ${ }^{3}$. Lacordaire n'en fut pas moins proche des idées de liberté, estimant nécessaire de préserver les acquis libéraux de la Révolution française de 1789.

Dans la même perspective de justice en tant que source de paix et d'équilibre, Montesquieu, dans son essai intitulé L'Esprit des lois (1748), souligne : « Une chose n'est pas juste parce qu'elle est loi. Mais elle doit être loi parce qu'elle est juste ». A ce titre, afin d'éviter les dangers de créer des règles sans chercher son effectivité, de ne pas exacerber des frustrations, notamment celles vécues par des personnes cherchant à s'intégrer dans une nouvelle société, Richelieu ${ }^{4}$ nous rappelle que : "Faire une loi et ne pas la faire exécuter, c'est autoriser la chose qu'on veut défendre » (Richelieu, 1947[1688]).

Plus récemment, Rocher (2004, p. 136) résume la situation de la façon suivante:

Tenter de comprendre l'effectivité du droit, c'est tout ensemble retracer la diversité de ses effets, voulus et involontaires, recherchés ou accidentels, directs et indirects, prévus et inattendus, sociaux, politiques, économiques ou culturels.

En somme, des moyens précis doivent être mis en place pour que le droit devienne réalité et réponde aux diverses attentes et besoins des sujets de droit. Parallèlement, lorsque considéré de manière holistique, le "soin » a plusieurs dimensions et nécessite l'élaboration d'un environnement où le dialogue est possible. Une savante combinaison de la littératie juridique et de la littératie en santé augmenterait la possibilité de créer cet espace de dialogue afin de maximiser les possibilités pour une personne d'accéder et de s'approprier son droit à la santé.

L'acquisition de la connaissance des droits implique aussi la compréhension de la culture juridique. Dans ce contexte, déjà en 1998, Bourdieu rappelait que d'une part que la connaissance est une arme et c'est ainsi que la domination consiste en la transmission du capital, qu'il soit économique, culturel ou social au sein du même cercle, des mêmes réseaux, souvent solidement tissés et qui contribuent ainsi à renforcer les situations inégalitaires (Boisvert, 2009; Bourdieu, 1998 et 2003).

Donc, en plus de s'assurer que les personnes connaissent leurs droits, elles doivent, en tant que sujet de droit, se les approprier pour être en mesure de les exercer de façon éclairée (Bates, 2006). Une telle littératie juridique contribuerait à l'amélioration des aptitudes d'une personne à exercer effectivement ses droits. S'agissant de la littératie en santé, des travaux émergent (Car, Lang, Colledge, Ung, et Majeed, 2011; Peerson et Saunders, 2009; European Center for Disease Prevention and Control, 2012; Simich, 2009). Bien que certains auteurs se penchent sur l'importance de la " conscientisation » juridique des citoyens (Hertogh, 2009; Silbey, 2005), l'articulation du concept de littératie juridique (tant au niveau des sciences sociales qu'au plan du développement de moyens juridiques concrets) doit se poursuivre afin d'assurer, de façon plus pérenne, la capacité à reconnaître la nature juridique d'un conflit et de savoir où trouver les sources d'information et de ressources d'accompagnement juridique nécessaires pour faire valoir ses droits (Manley-Casimir et Cassidy, 1986). De tels savoirs doivent être cultivés auprès des citoyens au-delà d'une formation juridique dans une école de droit. II s'agit d'un autre exemple de la responsabilité des intervenants concernant le renforcement d'une approche citoyenne des usagers tant dans le domaine de la santé que juridique.

\footnotetext{
2 Jean-Baptiste-Henri Dominique Lacordaire (1802-1861) était un religieux dominicain considéré comme un précurseur du catholicisme moderne, de la démocratie chrétienne et le restaurateur en France de son ordre.

${ }^{3}$ Extrait de la Quarante-cinquième conférence de Notre-Dame, 1972. Dans J. Carbonnier (dir.), Droit Civil, Vol. IV (p. 41). Paris : PUF

${ }^{4}$ Armand Jean du Plessis de Richelieu (1585 - 1642), cardinal-duc de Richelieu et duc de Fronsac, est un ecclésiastique, homme d'État français, pair de France et le principal ministre du roi Louis XIII. Réputé pour son habileté voire pour son caractère jugé retors, souvent critiqué pour sa fermeté intransigeante, il rénove la vision de la raison d'État et en fait la clé de voûte de ses méthodes de gouvernement et de sa conception de la diplomatie et de la politique.
} 


\section{Expérience migratoire et accès aux services de santé}

L'expérience d'immigration se trouve à l'intersection d'une diversité de déterminants sociaux de la santé. Lors d'une expérience d'intégration, l'accessibilité à un logement satisfaisant, à une éducation de base ou à des séances d'information sur son nouvel environnement, l'aisance dans la langue de la société d'accueil, le degré de précarité en emploi et le niveau de revenu, la qualité des réseaux sociaux et les rapports de genre deviennent des facteurs relativement importants selon les contextes de vie (Bowen, 2001; Oxman-Martinez et collab., 2005; Sen, 1993; Snow, 2008; Vissandjée, Apale, et Wieringa, 2009; Vissandjée et Battaglini, 2010). Ces expériences reliées à l'immigration agiraient ainsi tantôt comme source d'amélioration de la capacité de résilience aidant à réussir une nouvelle vie, tantôt comme source d'augmentation de certains facteurs de risque pour la santé (Ungar, 2006, 2008). Cette combinaison dynamique de facteurs exposerait de manière disproportionnée certaines personnes à des problèmes de santé, donnant lieu à diverses stratégies d'intégration, notamment dans les champs sociaux et économiques qui influeront sur d'autres déterminants de la santé.

Les expériences de santé et de maladie comportent de multiples dimensions biologiques et sociales. Elles sont influencées tant par les choix qui s'offrent aux personnes que par les ressources qui sont disponibles pour elles et leur sont accessibles. Les politiques et les pratiques en matière de santé jouent également un rôle dans la façon dont ces expériences sont vécues et leurs conséquences. Si ces expériences souvent singulières et leurs effets spécifiques sur la capacité à gérer sa propre santé, dont l'accès à des soins de santé de qualité, étaient prises en compte dans les politiques et dans les programmes sociaux et de santé, cela se traduirait par une sensibilité à la complexité des identités, des genres, des rapports de genre, des façons de faire et des visions et à leur capacité de transformation au gré des expériences. Or cette sensibilité n'est pas complètement acquise, notamment lors de l'élaboration, de l'implantation et de l'évaluation des programmes et des politiques dans le domaine de la santé et des services sociaux (Bates, Hankivsky et Springer, 2009; Vissandjée et Battaglini, 2010).

Une prise en compte plus systématique de la nature croisée des déterminants sociaux de la santé, des intersections de marqueurs d'identité, au-delà d'une liste de "sujets d'intervention " isolés - et notamment des questions de genre, d'appartenance ethnique et de migration - permettrait aux systèmes de soins et de santé de mettre en exergue les dynamiques de structuration sociale, les hiérarchies de pouvoir, les sphères d'inégalité et ainsi la nature complexe des défis d'accès aux soins de santé pour les personnes vulnérables (Dyck et McLaren, 2002; Krieger, Smith, Naishadham, Hartman, et Barbeau, 2005; Oxman-Martinez et collab., 2005; Raphael, 2006).

Il est dans ce cadre important de distinguer clairement la notion de sexe biologique de celle de genre, comme nous l'avons fait en introduction. Le concept de genre fait également référence à la façon dont une personne est perçue, selon qu'elle est une femme ou un homme, en tant qu'actrice dans une société donnée (Bates, Hankivsky et Springer, 2009; Hyman et Guruje, 2002; Johnson, Greaves et Repta, 2007; Vissandjée, Weinfeld, Dupéré, Abdool, 2001). Bien que les termes sexe et genre soient souvent utilisés de façon interchangeable, ils représentent des unités d'analyse distinctes. Ces termes différents exigent de s'attarder à la question du genre et des rapports de genre dans les programmes sociaux et de santé afin de mieux rendre compte de l'existence de certaines inégalités encore trop présentes dans l'accès à l'information et aux ressources. Une sensibilité pertinente à la différence entre les notions de sexe biologique et aux questions reliées au genre ne peut qu'enrichir l'accessibilité aux interventions, voire même augmenter leur efficacité.

L'expérience migratoire ne se présente généralement pas sous la forme d'un processus linéaire uniforme pour les femmes et les hommes. Cette trajectoire est construite à travers des expériences d'intégration à degrés variables (Johnson et Beaudet, 2013; Johnson, Greaves et Repta, 2007; Spitzer et Torres, 2008; Vatz-Laaroussi et Rachédi, 2002; Vissandjée et Battaglini, 2010). S'inscrire dans le prisme de la vulnérabilité exige de circonscrire les lieux possibles d'inégalités sociales ainsi que des conditions nécessaires à la justice en matière d'opportunités et de choix. Prendre en compte ces expériences de transition, notamment lors de la pratique clinique, ne peut qu'ouvrir à la sensibilisation des périodes de changement et des trajectoires de vie. Ces dernières représentent un processus complexe, étroitement liées à la signification donnée pour la personne qui vit ces transitions selon l'environnement dans lequel elle est plongée (Graham et Kelly, 2004; Meleis, Sawyer, Im, Hilfinger Messias et Schumacher, 2000; 
Sen et lyer, 2012). La santé des personnes immigrantes est le reflet de relations complexes et dynamiques entre les facteurs biologiques, psychologiques, sociaux et contextuels.

C'est dans une perspective similaire que Spitzer et Torres (2008), Ungar (2006, 2008), Walsh (2003) et Antonovsky (1992) ont inscrit leurs travaux visant à comprendre comment des facteurs tels que la résilience, la détermination et un sens de cohérence permettent aux familles vivant des expériences d'immigration de se mobiliser positivement, traverser des obstacles dans leurs trajectoires d'intégration, de vie et renforcer par le biais même les relations familiales et communautaires. II y a déjà fort longtemps que des auteurs, notamment Antonovsky en 1992, suggèrent la prise en compte des caractéristiques ou déterminants sociaux de la personne vivant ces expériences d'intégration et ce de façon particulière : l'âge, la différence marquée de statut social entre le pays d'origine et le pays d'arrivée, le statut d'immigration et le degré d'isolement. Antonovsky a ainsi proposé un cadre visant à mieux comprendre le rôle de facteurs de risque et de protection associé au stress d'intégration. Les conséquences sont associées au sens de cohérence qu'une femme et un homme attribue, de façon différenciée à ce type de stress, selon qu'ils sont prévisibles et gérables avec les ressources disponibles et accessibles.

\section{Vulnérabilité, accès aux soins de santé et pratique clinique}

Une perspective tenant compte du parcours de vie et rendant compte des expositions cumulatives " renseignera " donc plus adéquatement sur les facteurs de vulnérabilité comme de résilience. Une approche sensible aux intersections de la diversité exige en effet que l'on s'attarde aux caractéristiques profondément enracinées tout autant qu'aux facteurs de risque liés aux éléments structurels issus de certaines politiques d'immigration, notamment celles relatives aux ressources humaines et à la main d'œuvre, politiques parfois peu sensibles aux droits (Hankivsky, 2012).

Lors des interactions cliniques, la personne livre au soignant une histoire, un vécu d'une maladie qui est inscrite dans sa trajectoire et façonnée de ses représentations. C'est ce sentiment de désordre au moment du vécu tant par l'expérience de la maladie que celle relative à une identité qui se construit dans une nouvelle société qu'il s'agit pour le soignant de capter dans la narration, le partage qui aura lieu lors des interactions cliniques. En somme, les " intervenants » dans la bureaucratie juridique deviennent des "interprètes », des "traducteurs ", similairement aux intervenants de la santé dans leur impératif de donner une qualité des soins en présence de barrière linguistique (Battaglini, 2007; Bowen, 2005; Carnevale, Vissandjée, Nyland et Vinet-Bonin 2009; Leanza, 2005; Vissandjée et Maillet, 2007).

Par exemple, les obstacles d'accès à une indemnisation suite à une lésion musculo-squelettique subie par des travailleurs immigrants illustre de façon éloquente les jeux d'influence de l'environnement professionnel de la personne lésée (Gravel et collab., 2010). Gravel et ses collaborateurs font ressortir les séries de filtres de vulnérabilité subis par les travailleurs. De façon plus précise, on retrouve les freins exercés par les supérieurs et les pairs, les enjeux de pouvoir entre l'expertise et la contre-expertise relative à la lésion. Un exemple illustrant les interactions parfois convergentes, mais le plus souvent divergentes, des disciplines médicales et juridiques. Enfin, la hiérarchie administrative, tout en étant responsable de la compréhension des bonnes procédures à suivre par les réclamants, applique une certaine dynamique ne favorisant pas la personne lésée dans sa trajectoire de demande d'indemnisation (Azaroff, Levenstein, et Wegman, 2002). De ce point de vue, le rôle que jouent l'interprétariat et la médiation culturelle dans l'accès aux soins est crucial. Toutefois, il y a dans ces exigences normatives minimales une affirmation implicite qui va au-delà de la thèse habituelle qui s'adosse de l'argument d'universalité et d'égalité d'accès aux soins. En érigeant l'égalité en norme absolue, cette thèse fait des obligations d'échange avec des points de rencontre linguistique entre soignants et soignés une obligation institutionnelle et un droit de créance pour la personne nécessitant des soins.

Dans le cadre de la pratique clinique, ce sera la rencontre de deux personnes, deux cultures nécessitant un travail de prise en compte de ces trajectoires de vie. Alors que la personne livrera au soignant comment elle se sent, le soignant devra accepter de se décentrer, de remonter à rebours les expériences de vie dans leur contexte spécifique en tant femme, homme, immigrant en processus d'intégration et d'apprentissage des droits conférés par le pays d'accueil (Bischoff et collab., 2003). Ainsi, une pratique clinique auprès de personnes vulnérabilisées 
notamment par un accès limité à la langue du pays d'accueil exigera du soignant justement de considérer la diversité des expériences, de faire le lien entre les mondes tout en interprétant conceptuellement le discours des institutions et leurs pratiques aux soignés (Larchanché et Sargent, 2009).

Les obstacles de nature linguistique peuvent, parfois, apparaître comme une question secondaire de la pratique clinique. Mais en y regardant de plus près, cette apparence s'estompe et fait surgir les exigences normatives d'universalité des soins. Certes la personne nécessitant des soins ne peut accéder véritablement aux soins si des mécanismes institutionnels ne sont pas mis en place pour surmonter la barrière des langues. II nous faut cependant pénétrer plus avant dans la formulation de la question et il est même possible de l'élargir. C'est ainsi que la communication et le dialogue clinique relèvent de l'essence même de l'art de soigner et de la responsabilité clinique (Carnevale et collab., 2009). C'est en vertu de l'égalité d'accès aux soins, et davantage au nom même de l'adéquation par rapport aux règles de l'art et à l'éthique des soins que la nécessité des échanges doit être comprise. Posée ainsi, la question de l'interprétariat acquiert une nouvelle acuité. Nous situons cet impératif sur le terrain de l'éthique des soins (Vissandjee, Hemlin, Gravel, Roy, et Dupéré, 2005).

Plus précisément, Carnevale (1998) explique que l'éthique en soins infirmiers n'est pas définie exclusivement par un code d'éthique, car un code est seulement l'une des sources en soins infirmiers. Par exemple, les normes acceptées de pratique peuvent être définies par la façon dont les infirmières se comportent dans certaines situations données, et délimitent donc ce qui constitue une bonne pratique infirmière (Carnevale, 2007). La législation et la jurisprudence déterminent les normes légales pour la profession infirmière et la médecine (dont notamment, les impératifs du consentement libre et éclairé et la non-discrimination ${ }^{5}$ ). Ces normes informent également des impératifs pour l'éthique en soin infirmier et en médecine dans une juridiction. Or, il n'y a pas de normes éthiques explicites qui régissent les obligations des infirmières qui dispensent des soins en contexte de distance linguistique (Carnevale et collab., 2009).

\section{Investir dans le dialogue, pour une meilleure justice sociale}

Dans le cas de la justice comme dans celui de la santé, la rencontre donne lieu à des espaces de dialogue et de traduction sur diverses dimensions, s'ajoutant, pour les nouveaux arrivants, au défi de l'apprentissage des tenants et aboutissants de la société d'accueil. Une certaine harmonie de connaissances en matière d'accès à la justice et à des soins de qualité implique, en amont, un degré de résilience de la part de la personne pour qu'elle connaisse, comprenne et utilise à bon escient ses droits.

Rootman et ses collaborateurs ont défini la littératie de la façon suivante : " La capacité pour les individus de repérer, comprendre, évaluer et communiquer de l'information pour être capables de composer avec les divers contextes de santé afin de promouvoir la santé tout au long de leur vie. »(2006, p. 81). La littératie en santé est donc d'autant plus importante pour les personnes immigrantes, et plus spécifiquement pour les femmes, que leur accès aux services de soins de santé peut être compromis par la présence de barrières linguistiques et culturelles (Bischoff et collab., 2003; Bowen, 2005; Fortin et Le Gall, 2007; Rootman, Kaszap, et Frankish, 2006; Simich, Maiter, et Ochocka, 2009; Vatz-Laaroussi et Rachédi, 2002). Les effets de ces barrières sont souvent manifestes dès le premier contact - ou tentative de contact - auprès des intervenants des services de santé (Munoz et Kapoor-Kohli, 2002). Le degré de distance entre les personnes soignantes et soignées est un facteur décisif dans les rencontres cliniques, les barrières se révélant à mesure que le soin se complexifie. Il a été documenté également que des situations d'incompréhension dans des interactions de soins, notamment en situation d'urgence ou de crise, ont contribué à des décès prématurés qui auraient pu être évités ${ }^{6}$.

\footnotetext{
${ }^{5}$ Exemples de jurisprudence aux États-Unis (Schloendorff v. Society of New York Hospitals, 1914); Exemple au Québec (Mlle Bordier c. S, 1934); Exemple en France (Cass. Civ. Le, 1936)

${ }^{6}$ Bureau du Coroner du Québec : Dossiers traités par le Dr Jacques Ramsay : 127466, 127752, 140155, 151404 et 151407 et le dossier 147166 par Me Catherine Rudel-Tessier.
} 
Cette capacité de s'exprimer et d'être entendu s'inscrit dans le processus de prise de contrôle sur les droits de la personne immigrante, que ce soit dans le domaine de la santé ou de la justice. Permettre l'expression de ces acquis de connaissance par le citoyen exige de la part des intervenants, qu'ils soient dans le domaine de la santé ou dans celui de la justice, de reconsidérer les normes et règles de conduite fondées sur des savoirs spécialisés sans toutefois disqualifier les savoirs informels (Fortin et Le Gall, 2007; Massé et Saint-Arnaud, 2003; Simich et collab., 2009). Plusieurs stratégies peuvent être mises en œuvre par les prestataires et les gestionnaires de la santé afin de contribuer à renforcer ce niveau de littératie des personnes, par le biais de formations de part et d'autre, d'outils, de lignes directrices sur l'intervention en contexte de distances culturelle et linguistique, également des interventions personnalisées s'inscrivant dans les particularités des trajectoires d'immigration des femmes et des hommes (Simich et collab., 2009).

Les acteurs dans le domaine du droit et de la santé ne peuvent donc faire autrement que de se sensibiliser aux expériences différenciées, même si subjectives, des femmes et des hommes à titre d'experts de leur propre vie (Constitution de l'OMS, 1946; voir Fortin-Pellerin, 2006; Ninacs, 2008; Vissandjée et Maillet, 2007). De reconnaître cette expertise, d'encourager son expression et cela dans sa variabilité expérientielle, entre autres relativement aux rapports sociaux et de genre, contribue à cette nécessaire et incontournable conciliation des savoirs et des pratiques vers des alliances thérapeutiques plus efficaces (Fortin et Le Gall, 2007; Vissandjée et Dallaire, 2002). Dans cette perspective, les expériences de vie devraient être examinées, prenant en compte les identités en mouvance, les facteurs de risque et de fragilité, les capacités de résilience ancrées dans des relations sociales complexes interagissant les unes avec les autres. Ainsi, toute situation de vulnérabilité peut évoluer, puisqu'elle se trouve sans cesse à la croisée d'identités, de trajectoires, d'un territoire social à un autre où divers espaces et ressources se conjuguent. Ceci crée un contexte où des rapports sociaux d'inclusion et d'exclusion en évolution constante. Dans cette perspective de trajectoire d'intégration spécifique aux hommes et aux femmes, les rapports sociaux se constituent et se reconstituent constamment où chacun et chacune n'est pas toujours un "sujet de droit » dans une perspective d'équité. (Aubert, 2007; McAll et collab., 1997).

Il est opportun de revenir aux mots laissés par Rousseau, en 1871, dans son Discours sur l'origine des langues, avec une perspective adoptée par nos contemporains, nommément l'importance de la pratique réflexive (Bulman et Schutz, 2008; Lafortune, 2012; Paget, 2001; Turcotte, 2012). II est nécessaire, que ce soit dans le domaine de la santé ou du droit, de " porter sa vue au loin ". Ces mots de Rousseau, cités en exergue, nous rappellent à juste titre l'importance d'observer et d'apprécier les différences entre les êtres humains pour connaître l'Homme dans sa spécificité. Ces quelques paroles ouvraient, deux siècles avant Lévi-Strauss, la voie à l'examen du contexte de vie dans sa complexité différentielle. II s'agit d'observer et de tenir compte des différences pour en découvrir les richesses et une certaine proximité. Il s'agit aussi de réfléchir, de voir, de se voir, de se penser et, corollairement, tourner, se tourner vers soi-même, faire tourner, à mobiliser de manière efficace l'ensemble des ressources tant internes qu'externes que nous détenons en tant qu'intervenants mais aussi que possèdent les femmes et les hommes requérant un accès à des services et aux soins de santé ${ }^{7}$. Paul Éluard résume cette quête vers un ordre plus structuré et inclusif par l'une de ses fameuses citations : «II nous faut peu de mots pour exprimer l'essentiel; il nous faut tous les mots pour le rendre réel $\Perp^{8}$.

Une reconnaissance des zones de force de la personne nécessitant des services et des soins devient cohérente non seulement avec les exigences de pratique requises de la part de la personne "prestataire " d'un service ou d'un soin, mais également à un contexte sensible à la justice sociale vers l'effacement des inégalités sociales (BrydonMiller, Maguire, et Mclntyre, 2004; Hankivsky, 2005; Reid, Pederson, et Dupéré, 2006; Roy, Frechet, et Savard, 2008; Saillant et Gagnon, 1999; Schulz et Mullings, 2006; Vissandjée et Hyman, 2011). Interpeller la subjectivité des acteurs sociaux, c'est leur donner une voix au-delà de la représentation sociale et de l'influence des valeurs culturelles.

\footnotetext{
${ }^{7}$ Inspiration de la préface écrite par M. Bettens pour L. Lafortune dans Stratégies réflexives-interactives pour le développement de compétences : La formation en éducation et en santé, 2012, Presses de l'Université du Québec.

${ }^{8}$ Citation de Eugène Émile Paul Grindel, dit Paul Éluard, poète français (1895-1952) dans Avenir de la poésie (1937).
} 
Ce rapport égalitaire repose sur un processus social dont la responsabilité est partagée entre la personne immigrante et la société d'accueil et qui vise l'intégration au détriment de la marginalisation. Comme nous l'avons démontré plus haut, en contexte migratoire, les possibilités de recours à des soins de santé ou autres ressources (légales) peuvent être compromises, si au départ, les personnes ne connaissent pas leurs droits à ces accès.

Une telle perspective vise à éviter de cantonner les personnes vivant des expériences d'immigration à une destinée fixée dans la vulnérabilité. Afin d'agir sur les rapports inégalitaires issus des situations de vulnérabilité, il faut donc saisir et déconstruire les pratiques d'inclusion et d'exclusion dans l'accès à la santé et à la justice. Pour ce faire, les marqueurs d'identités doivent être réexaminés à travers différentes frontières et sa propre individualité en tant que prestataire en santé ou expert en justice, en tant qu'êtres ayant une trajectoire et un contexte de vie unique à tout un chacun et chacune.

\section{Adapter les pratiques : pour la reconnaissance de l'expérience de l'autre}

Si les questions relatives à l'importance de la prise en compte de l'intersection entre des déterminants tels que les rapports de genre, l'appartenance ethnique et les expériences d'immigration peuvent sembler peu palpables, elles n'en sont pas moins essentielles pour le succès de l'application des programmes et politiques dans le domaine des soins de santé. Les établissements de santé et de services sociaux, les décideurs et les prestataires doivent remettre en question leur compréhension des facteurs modulant notamment la capacité des femmes immigrantes à promouvoir leur santé, à se maintenir en santé et à gérer cette dernière en cas de maladie et cela afin d'intervenir sur la nature croisée de ces facteurs.

Des analyses plus fines des concepts en jeu et un débat plus poussé sur les méthodes utilisées pour mesurer ces concepts et variables évoluant en intersection sont requises (Rummens, 2002; Spitzer, 2005; Vissandjée et Dupéré, 2000). À ce sujet, les approches de recherche et les stratégies d'intervention se devront de créer des espaces plus pertinents, sécuritaires et plus inclusifs (Hankivsky, 2005; Hankivsky, 2012; Reid et collab. 2006).

Le rapport de la Commission des déterminants sociaux de la santé (OMS, 2008) souligne que les inégalités sociales peuvent être redressées si des efforts de société sont effectivement mis en place. Plus que jamais, il est nécessaire d'agir sur les fondements mêmes des structures pour transformer les rapports sociaux. Dans une ère d'incertitude et de croissance de l'écart entre les extrêmes, notre société ne peut faire l'économie de renforcer les potentiels qu'elle recèle en passant par un système plus efficace d'accès à l'information, une condition nécessaire de succès du droit à l'accès (Beauchemin, 2008; Hirsh, 2006; Labesse, 2009; Thérien, 2009). Afin de permettre le développement de dialogues assurant la mise en œuvre du droit aux soins de santé de qualité, il est important que les nouveaux arrivants reçoivent plus d'information sur leurs droits. De plus, les intervenants du système de santé qui les accueillent doivent aussi être informés de la condition des nouveaux arrivants et de leurs droits. Un important partage d'informations doit avoir lieu, non seulement dans le cadre de formations mais surtout à travers des espaces de dialogues permettant une véritable rencontre.

En permettant un lieu d'expression aux arguments expérientiels, une intégration et une reconnaissance de la légitimité des savoirs d'expérience en plus des savoirs experts, un « réel » espace de dialogue s'instaurera (Labesse, 2009; Mantoura, Gendron, et Potvin, 2007). De tels dialogues devraient mener vers des effets tangibles sur le " contexte » de vie des personnes immigrantes vivant dans des situations de vulnérabilité, sur la réponse à leurs besoins essentiels et sur la parité citoyenne dans l'exercice des rôles sociaux. Combattre les inégalités sociales est certainement une affaire de justice, mais c'est avant tout une question de volonté, une question de promouvoir une manifestation citoyenne positive. 


\section{Références bibliographiques}

Anand, S., Peter, F. et Sen, A. (2004). Public Health, Ethics, and Equity. Oxford / New York : Oxford University Press. Antonovsky, A. (1992). Can attitudes contribute to health? The Journal of Mind-Body Health, 8, 33-49.

Aubert, J.L. (2007). Introduction au droit. Paris : Presses universitaires de France.

Azaroff, L. S., Levenstein, C. et Wegman, D. H. (2002). Occupational Injury and Illness Surveillance: Conceptual Filters Explain Underreporting. American Journal of Public Health, 92(9), 1421-1429.

Bulman, C. et Schutz, S. (2008). Reflective Practice in Nursing (4 éd.). Oxford : Blackwell Publishing.

Bates, K. (2006). Du texte au sujet en action : les défis de l'accès à la justice en Inde. Cahiers d'anthropologie du droit, 6, 75-96.

Bates, K. (2007). Trajectoire ethnographique : du dialogue au devoir de synthèse. Altérités, 7(1), 21-37.

Bates, L., Hankivsky, O. et Springer, K. (2009). Gender and health inequities: a comment on the final report of the WHO commission on the social determinants of health. Social Science and Medicine, 69(7), 1002-1004.

Battaglini, A. (2007). L'intervention de première ligne à Montréal auprès des personnes immigrantes : Estimé des ressources nécessaires pour une intervention adéquate. Québec : Direction de la santé publique.

Beauchemin, J. (2008). Vulnérabilité et crise du politique. Dans V. Châtel et S. Roy (dir.), Penser la vulnérabilité : visages de la fragilisation du social (p. 51-64). Québec: Presses de l'Université du Québec.

Bischoff, A., Bovier, P. A., Rrustemi, I., Gariazzo, F., Eytan, A. et Loutan, L. (2003). Language Barriers Between Nurses and Asylum Seekers: Their Impact on Symptom Reporting and Referral. Social Science and Medicine, 57(3), 503-512.

Boisvert, R. (2009). Les inégalités dans l'arène. Développement social, 10(2), 44-45.

Bouchard, L. (2003). Conseil canadien de développement social, L'inclusion sociale et la santé des Canadiens. Ottawa : Conseil canadien de développement social.

Bourdieu, P. (1998). La domination masculine. Paris : Seuil.

Bourdieu, P. (2003). L'objectivation participante. Actes de la recherche en sciences sociales, 150, 43-57.

Bowen, S. (2001). Barrières linguistiques dans l'accès aux soins de santé. Ottawa : Santé Canada.

Bowen, S. (2005). Development of a coordinated response to addressing language barriers within the Winnipeg Regional Health Authority. Winnipeg: Winnipeg Regional Health Authority.

Brydon-Miller, M., Maguire, P. et Mclntyre, A. (2004). Traveling companions feminism, teaching, and action research. Westport, $\mathrm{CN}$ : Praeger.

Santé Canada, (2000). Les déterminants sociaux de la santé. Ottawa : Santé Canada.

Car, J., Lang, B., Colledge, A., Ung, C. et Majeed, A. (2011). Interventions for enhancing consumers' online health literacy [en ligne]. Cochrane database of systematic reviews, 6, n CD007092.

Carnevale, F. (1998). The utility of futility: the construction of bioethical problems. Nursing ethics, 5(6), $509-517$.

Carnevale, F. (2007). the birth of tragedy in pediatrics: a phronetic conception of bioethics. Nursing ethics, 14(5), 571-582.

Carnevale, F., Vissandjée, B., Nyland, A. et Vinet-Bonin, A. (2009). Ethical Considerations in Cross-Linguistics Nursing. Nursing ethics, 16(6), 813.

Carrier, A. (2008). Le droit aux services d'ergothérapie en CLSC : entre légalité et légitimité. Sherbrooke : Université de Sherbrooke.

Conley, J. et O'Barr, W. (1998). Just Words : Law, Language, and Power. Chicago : University of Chicago Press.

Alterstice-Revue Internationale de la Recherche Interculturelle, vol. 3, $n^{\circ} 1$ 
Crenn, C. (2000). Une consultation pour les migrants à l'hôpital. Hommes et migrations (1225), 39-45.

Cooper, H. (2002). Investigating socio-economic explanations for gender and ethnic inequalities in health. Social Science and Medicine, 54(5), 693-706.

Dembe, A., Belton, S., Himmelstein, J. et Workers' Compensation Health Initiative. (2003). Improving workers' compensation medical care : a national challenge. Beverly Farms, MA : OEM Press.

Dion, M. et Breton, G. (1997). L'éthique gouvernementale. Saint-Laurent : Fides.

Dyck, I. et McLaren, A. (2002). Becoming Canadian? : Girls, home and school and renegotiating feminine identity. Vancouver: Vancouver Centre of Excellence.

European Center for Disease Prevention and Control. (2012). A rapid évidence review of interventions for improving health literacy: insight into health communication. Stockholm : ECDC.

Evans, R., Barer, M. et Marmor, Theodore R. (1996). Être ou ne pas être en bonne santé : biologie et déterminants sociaux de la maladie. Montréal : Presses de l'Université de Montréal.

Fortin-Pellerin, L. (2006). Contributions théoriques des représentations sociales à l'étude de l'empowerment. Journal international sur les représentations sociales, 3(1), 57-67.

Fortin, S. et Le Gall, J. (2007). Néonatalité et constitution des savoirs en contexte migratoire : familles et services de santé. Enfances, Familles, Générations, 6, 16-37.

Graham H., Kelly, M. P. (2004). Health Inequalities: concepts, frameworks and policy. London : Health Development Agency.

Gravel, S., Brodeur, J.-M., Champagne, F., Lippel, K., Patry, L., Boucheron, L., Fournier, M. et Vissandjée, B. (2007). Critères pour apprécier les difficultés d'accès à l'indemnisation des travailleurs immigrants victimes de lésions professionnelles [en ligne]. Perspectives interdisciplinaires sur le travail et la santé, 8(2). http://pistes.revues.org/3025

Gravel, S. et Régie régionale de la santé et des services sociaux de Montréal-Centre. Unité écologie humaine et, sociale. (2000). Culture, santé et ethnicité : vers une santé publique pluraliste. Montréal: Régie régionale de la santé et des services sociaux de Montréal-Centre.

Gravel, S., Vissandjee, B., Lippel, K., Brodeur, J-M., Patry, L. et Champagne, F. (2010). Ethics and the Compensation of Immigrant Workers for Work-Related Injuries and IIInesses. Journal of Immigrant and Minority Health, 12(5), 707-714.

Gulliford M., Figueroa-Munoz, J., Morgan, M., Hughes, D., Gibson, B., Beech, R. et Hudson, M. (2002). What does "access to health care" mean? Journal of Health Services Research and Policy, 7, 186-188.

Hankivsky, O. (2005). Gender vs. diversity mainstreaming: a preliminary examination of the role and transformative potential of feminist theory. Canadian Journal of Political Science, 38(4), 977-1001.

Hankivsky, O. (2012). Gender and health : relational, intersectional, and biosocial approaches. Social Science and Medicine, 74, 1661-1666.

Hertogh, M. (2009). What's in a handshake? Legal equality and legal consciousness in the Netherlands. Social and Legal Studies, 18(2), 221-239.

Hirsh, M. (2006). Les formes modernes de la pauvreté. Dans P. Rosanvallon (dir.), Nouvelle critique sociale (p. 7181). Paris : Seuil.

Hubert, A. (2004). Corps de femmes sous influence : questionner les normes. Paris : Observatoire Cniel des habitudes alimentaires.

Hyman I., Guruge S. (2002). A review of theory and health promotion strategies for new immigrant women. Canadian Journal of Public Health, 93, 183-187. 
Johnson, J. et Beaudet, A. (2013). Sex and gender reporting in health research: why Canada should be a leader. Canadian Journal of Public Health, 104(1), e80-e81.

Johnson, J., Greaves, et L., Repta, R. (2007). Better science with sex and gender: a primer for health research. Vancouver: Women's Health Research Network.

Karlsen, S. et Nazroo, J. Y. (2002). Agency and structure: the impact of ethnic identity and racism on the health of ethnic minority people. Sociology of Health and IIIness, 24(1), 1-20.

Krieger, N., Smith, K., Naishadham, D., Hartman, C. et Barbeau, E. M. (2005). Experiences of discrimination: validity and reliability of a self-report measure for population health research on racism and health. Social science and medicine, 61(7), 1576-1596.

Labesse, M. (2009). L'égalité hommes-femmes : une joute essentielle au bien-être de toutes et de tous. Développement social, 10(2), 14-15.

Lafortune, L. (2012). Des stratégies réflexives-interactives pour le développement de compétences : la formation en éducation et en santé. Presses de I'Université du Québec.

Lajoie, A. (2004). Surdétermination. Dans Lajoie, A., MacDonald, R.A., Janda, R. et Rocher, G. (dir.), Théories et émergence du droit : pluralisme, surdétermination et effectivité. Montréal : Éditions Thémis.

Lalonde, L. (2011). Les « Lois Éthiques », un défi pour le droit. Revue Éthique publique, 12(2), 1-19.

Larchanché, S. et Sargent, C. (2009). Sur le pas de l'anthopologie médicale en France : la maturation d'une approche sur la place de la culture dans les soins de santé mentale. TransFaire et Cultures, 1(1), 100.

Lawrence, K. et Rieder, A. (2007). Methodologic and ethical ramifications of sex and gender differences in public health research. Gender Medicine, 4(Suppl. B), S96-S105.

Le Blanc, M., Raynault, M. et Lessard, R. (2011). Les inégalités sociales de santé à Montréal. Le chemin parcouru. (Rapport du directeur de santé publique 2011). Montréal : Agence de la santé et des services sociaux de Montréal.

Le Bossé, Y. (2004). De " I'habilitation » au « pouvoir d'agir » vers une définition plus circonscrite de la notion d'empowerment. Nouvelles pratiques sociales, 16(2), 30-51.

Leanza, Y. (2005). Roles of community interpreters in pediatrics as seen by interpreters, physicians and researchers. Interpreting, 7(2), 167-192.

Legault, G. et Rachédi, L. (2008). L'intervention interculturelle. Montréal : G. Morin/Chenelière éducation.

Macall, C. (2012). Au-delà du préjugé : trajectoires de vie, pauvreté et santé. Québec : Presses de l’Université du Québec.

Manley-Casimir, M. et Cassidy, W. (1986). Legal literacy : towards a working definition (rapport). Ottawa : Institut canadien d'information juridique.

Mantoura, P., Gendron, S. et Potvin, L. (2007). Participatory research in public health: Creating innovative alliances for health. Health and Place, 13(2), 440-451.

Massé, R. et Saint-Arnaud, J. (2003). Éthique et santé publique : enjeux, valeurs et normativité. Québec : Presses de l'Université Laval.

Mateu, J., Reynier, M. et Vialla, F. (2000). À la croisée des regards : Regards croisés sur le genre. Paris : Les Études hospitalières.

McAll, C., Tremblay, L. et Le Goff, F. (1997). Proximité et distance : les défis de communication entre intervenants et clientèle multiethnique en CLSC. Montréal : Éditions Saint-Martin.

Meleis, A. , Sawyer, L., Im, E., Hilfinger Messias, D. et Schumacher, K. (2000). Experiencing transitions: an emerging middle-range theory. Advances in nursing science, 23(1), 12-28. 
Mertz, E. (2007). The language of law school learning to "Think Like a Lawyer". Oxford: Oxford University Press.

Mikkonen, J. et Dennis, R. (2011). Les déterminants sociaux de la santé : les réalités canadiennes. Toronto : École de gestion et de politique de la santé de l'Université York.

Munoz, M. et Kapoor-Kohli, A. (2002). Les barrières de langue, comment les surmonter en contexte clinique. Le Médecin du Québec, 42(2), 45.

Ninacs, W. (2008). Empowerment et intervention : développement de la capacité d'agir et de la solidarité. Québec : Presses de l'Université Laval.

Organisation internationale du travail. (2008). Déclaration de l'Organisation internationale du travail sur la justice sociale pour une mondialisation équitable [en ligne].

http://www.ilo.org/global/resources/WCMS_099767/lang--fr/index.htm

Organisation mondiale de la santé (OMS). (2008). Combler le fossé en une génération : instaurer l'équité en santé en agissant sur les déterminants sociaux de la santé [en ligne].

http://www.who.int/social_determinants/final_report/media/csdh_report_wrs_fr.pdf

Oxman-Martinez, J., Hanley, J., Lach, L., Khanlou, N., Weerasinghe, S. et Agnew, V. (2005). Intersection of canadian policy parameters affecting women with precarious immigration status: a baseline for understanding barriers to health. Journal of Immigrant and Minority Health, 7(4), 247-258.

Paget,T. (2001). Reflective practice and clinical outcomes : practitioners' views on how reflective practice has influenced their clinical practice. Journal of Clinical Nursing, 10, 204-214.

Peerson, A. et Saunders, M. (2009). Men's Health Literacy: Advancing Evidence and Priorities. Critical Public Health, 19(3-4), 441-456.

Pietrantonio, L. (2000). Une dissymétrie sociale : rapports sociaux majoritaire/minoritaires. Cahiers d'études bastidiennes, 29-30, 151-176.

Raphael, D. (2006). Les inégalités de santé au Canada : faible préoccupation, actions insatisfaisantes, succès limités. Dans M. O’Neil, A. Pederson, S. Dupéré et I. Rootman (dir.), Promotion de la santé au Canada et au Québec : Perspectives critiques (p. 138-159). Québec : Presses de l'Université Laval.

Reid, C., Pederson, A. et Dupéré, S. (2006). Diversité et promotion de la santé : I'utilité des travaux sur la santé des femmes et sur la théorie intersectionnelle. Dans M. O’Neil, A. Pederson, S. Dupéré et I. Rootman (dir.), Promotion de la santé au Canada et au Québec: Perspectives critiques (p. 98). Québec: Presses de l'Université Laval.

Relis, T. (2009). Perceptions in litigation and mediation : lawyers, defendants, plaintiffs, and gendered parties. Cambridge / New York : Cambridge University Press.

Richelieu (Cardinal de) (1947 [1688]). Testament politique (édition critique par L. André). Paris : R. Laffont.

Rocher, G. (2004). L'effectivité du droit. Dans A. Lajoie (dir.), Théories et émergence du droit : pluralisme, surdétermination et effectivité (p. 133-149). Montréal : Éditions Thémis.

Rootman, I., Kaszap, M. et Frankish, J. (2006). La littératie en santé : un concept en émergence. Dans O’Neil, M, Pederson, A., Dupéré, S. et Rootman, I. (dir.), Promotion de la santé au Canada et au Québec : Perspectives critiques (p. 87). Québec: Presses de I'Université Laval.

Rousseau, J.-J. (1781). Essai sur l'origine des langues. Paris : Édition A. Berlin.

Roy, M., Frechet, G. et Savard, F. (2008). Le Québec, à l'avant-garde de la lutte contre la pauvreté au Canada. Policy Option Montreal, 29(8), 57-61.

Rummens, J. (2002). Redefining the Canadaian demos: towards a transcultural citizenship charter. Thèmes canadiens, février(édition spéciale), 15. 
Saillant, F. et Gagnon, E. (1999). Vers une anthropologie des soins. Présentation. Anthropologie et sociétés, 23(2), 514.

Schulz, A. et Mullings, L. (2006). Gender, race, class, and health: intersectional approaches. San Francisco, CA : Jossey-Bass.

Sen A. (1993). Capability and well-being. Dans M. Nussbaum et A. Sen, Quality of life (p. 30-53). Oxford : Clarendon Press.

Sen, A. (2000a). Repenser l'inégalité. Paris : Seuil.

Sen A. (2000b). Social exclusion: concept, application and scrutiny. Dans A. Sen, Social Development (p. 7-23). Office of Environment and Social Development, Asian Development Bank.

Sen, G. et lyer, A. (2012). Who gains, who loses and how : Leveraging gender and class intersections to secure health entitlements', Social Science and Medicine, 74, 1802-1811.

Silbey, S. S. (2005). After Legal Consciousness. Annual Review of Law and Social Science, 1(1) : 323-368.

Simich, L. (2009). Health Literacy and immigrant populations. Ottawa : Agence de santé publique du Canada.

Simich, L., Maiter, S. et Ochocka, J. (2009). From social liminality to cultural negotiation : transformative processes in immigrant mental wellbeing. Anthropology and medicine, 16(3), 253-266.

Snow, R. C. (2008). Sex, gender, and vulnerability. Global Public Health, 3(Supp. 1), 58-74.

Spitzer, D. L. (2005). Engendering health disparities. Revue canadienne de santé publique, 96(Supp. 2), S78-S96.

Spitzer, D. et Torres, S. (2008). Gender-based barriers to settlement and integration for live-in-caregivers: A review of the literature. CERIS Working Paper.

Thérien, F. (2009). Réduire les inégalités de richesse : une stratégie complémentaire de lutte à la pauvreté. Développement social, 10(2), 26.

Tingen, C., Kim, A., Wu, P. et Woodruff, T. (2010). Sex and sensitivity: the continued for sex-based biomedical research and implementation, Women's Health, 6(4), 511-516.

Turcotte, A. (2012). Processus de facilitation d'une pratique réflexive, de type co-développement, auprès d'infirmières pivots en oncologie et son influence sur leur pratique professionnelle. (Thèse de doctorat, Université de Montréal). https://papyrus.bib.umontreal.ca/xmlui/handle/1866/8580

Ungar, M. (2006). A thicker description of resilience. International Journal of Narrative Therapy and Community Work, 3-4, 89-96.

Ungar, M. (2008). Resilience across cultures. British Journal of Social Work, 38, 218-235.

Vatz-Laaroussi, M. et Rachédi, L. (2002). Femmes, mères et immigrantes : des enjeux de société et un appel au féminisme. Dans F. Descarries et C. Corbeil (dir.), Espaces et temps de la maternité (p. 431-455). Montréal : Éditions du remue ménage.

Vissandjée, B. et Dupéré, S. (2000). Culture, migration et enquête : défis incontournables. Journal of International Migration and Integration, 1(4), 477.

Vissandjée, B., Weinfeld, M., Dupéré, S. et Abdool, S. (2001). Sex, gender, ethnicity, and access to health care services: research and policy challenges for immigrant women in Canada. Revue de l'intégration et de la migration internationale, 2(1), 55-75.

Vissandjée, B. et Dallaire, M. (2002). Partenariat de recherche en mlieu plurietnique : des femmes immigrantes s'impliquent. Revue canadienne de santé publique, 93(6), 457.

Vissandjée, B., Hemlin, I., Gravel, S., Roy, S. et Dupéré, S. (2005). La diversité culturelle montréalaise: une diversité de défis pour la santé publique. Santé publique [Vandoeuvre-lès-Nancy, France], 17(3), 417-428.

Alterstice-Revue Internationale de la Recherche Interculturelle, vol. 3, $n^{\circ} 1$ 
Vissandjée, B. et Maillet, L. (2007). L'empowerment et l'expérience de l'immigration au Canada : multiples déterminants dans une réalité complexe. Dans H. Dorvil (dir.), Théories et méthodologies de l'intervention sociale. Problèmes sociaux (p. 143-162). Québec : Presses de l'Université du Québec.

Vissandjée, B., Apale, A. et Wieringa, S. (2009). Exploring social capital among in the context of migration: engendering the public policy debate. Dans V. Agnew (dir.), Racialized migrant women in Canada. Essays on health, violence, and equity (p. 187-203). Toronto : University of Toronto Press.

Vissandjée, B. et Battaglini, A. (2010). Santé des femmes : À la croisée des questions de genre, ethnicité et migration. Dans A. Battaglini (dir.), Les services sociaux et de santé en contexte pluriethnique (p. 277-292). Anjou : Éditions St-Martin.

Vissandjée, B. et Hyman, I. (2011). Preventing and managing diabetes: At th intersection of gender, ethnicity and migration. Dans O. Hankivsky (dir.), Transformational promise of intersectionality as a research paradign for improving the understanding of an response to diversity in health and illness (p. 257-273). Vancouver: University of British Columbia Press.

Walsh, F. (2003). Family resilience: A framework for clinical practice. Family Process, 42, 1-18.

Zanchetta, M. et Poureslami, I. (2006). Littératie en matière de santé dans la réalité des immigrants, sur le plan de la culture et de la langue. Revue canadienne de santé publique, 97(Supp. 2), S28-S33. 